\title{
RAÚL N. MARTÍNEZ CROVETTO: LOS ALBORES DE LA ETNOBOTÁNICA EN LA ARGENTINA. INTRODUCCIÓN A ESTUDIOS ETNOBOTÁNICOS V
}

\author{
ANALIA PIRONDO ${ }^{1}$ \& HÉCTOR A. KELLER ${ }^{1}$
}

\begin{abstract}
Summary: Pirondo, A \& H. A. Keller, 2012. Raúl N. Martínez Crovetto: the dawns of the Ethnobotany in Argentina. Introduction to Ethnobotanical Studies V. Bonplandia 21(2): 101-107.

Raúl Nereo Martínez Crovetto (1921-1988) went a versatile intellectual, an outstanding person, worthy of remember. In his long trajectory as a scientist, it deserves underline his inspiration that took him to introduce diverse topics in his publications, by leaving his own impression through the different contributions that he carried out. From the wide range of investigations that he made, we want stand out those related with topics and problems of the regional ethnobotany, informing on some theoretical and methodological perspectives done for the author in his investigations. Within field notes of Martínez Crovetto we found a broad list of names and uses of plants unpublished from his fieldwork with the Guarani Mbya in Misiones. He was director of Bonplandia between 1961-1983. This time, the journal present that work in the format of the series "Estudios etnobotánicos" created by Martinez Crovetto.
\end{abstract}

Key words: Ethnobotanical studies, Martínez Crovetto, Argentina

Resumen: Pirondo, A \& H. A. Keller. 2012. Raúl N. Martínez Crovetto: los albores de la Etnobotánica en la Argentina. Introducción a Estudios Etnobotánicos V. Bonplandia 21(2): 101107.

Raúl Nereo Martínez Crovetto (1921-1988) fue un intelectual polifacético, una persona destacada, digna de recordar. En su larga trayectoria como científico, merece subrayarse la inspiración que lo llevó a introducir diversos temas en sus publicaciones, dejando su impronta propia a través de los diferentes aportes que realizó. De la amplia gama de investigaciones realizadas, queremos destacar aquí las relacionadas con temas y problemas de la etnobotánica regional, para informar sobre algunas de las perspectivas teóricas y metodológicas aplicadas por el autor en sus investigaciones. Entre las notas de campo de Martínez Crovetto encontramos una amplia lista inédita de fitónimos y usos de plantas provenientes de su trabajo de campo con los guaraníes Mbya de la provincia de Misiones. En esta edición de Bonplandia, de la que además fuera director en el periodo 1961-1983, se las dará a conocer, siguiendo el formato de la serie "Estudios etnobotánicos" creada por Martínez Crovetto.

Palabras clave: Estudios etnobotánicos, Martínez Crovetto, Argentina

\section{Introducción}

El botánico estadounidense John William Harshberger acuñó por primera vez la palabra "etnobotánica" para referirse al estudio arqueológico de las plantas usadas por los pueblos aborígenes" (Ford, 1978). Con el tiempo, esta definición se ha ido ampliando, incluyendo a poblaciones actuales y no solo aborígenes. Además debido a los múltiples

${ }^{1}$ Instituto de Botánica del Nordeste, UNNE-CONICET, Corrientes, Argentina. E-mail: analiapirondo@hotmail. com,hectorkeller@hotmail.com 
enfoques que puede abordar esta disciplina, se ha vuelto marcadamente interdisciplinaria. Es así que en la actualidad existe un consenso generalizado en definir la etnobotánica como el estudio de las interrelaciones, dinámicas, que tienen lugar entre los vegetales y el hombre (Albuquerque, 2007).

En la Argentina, resulta difícil definir un momento preciso para el inicio de esta disciplina (Parodi, 1961). No obstante, podemos decir que los estudios de los misioneros jesuitas del siglo XVIII (Cabrera, 1979) constituyen un esbozo de aproximación etnobotánica. Asimismo, se puede considerar un trabajo de tipo etnobotánico a la reconocida obra "Plantae Diaphoricae" de fines del siglo XIX, primer inventario de las plantas usuales realizado por Hieronymus (1882). En ese contexto, siguieron los aportes de Domingo Parodi (1886), Eduardo Matoso (1893), Nicolás Rojas Acosta (1905, 1913); Juan A. Domínguez (1928); Lorenzo R. Parodi (1934, 1935); Arturo Ragonese y Raúl N. Martínez Crovetto (1947), entre otros.

En la actualidad, en nuestro país, la etnobotánica tiene un lugar definido, con trabajos de diversos autores reconocidos a nivel nacional e internacional. No obstante, no fue así en los años en los que el Ing. Agr. Raúl Nereo Martínez Crovetto (1921-1988) se aventuró a difuminar la frontera entre lo botánico y lo antropológico, para enfocarse en la realización de trabajos claramente interdisciplinarios, por lo cual se lo puede considerar hoy como uno de los fundadores de la etnobotánica en nuestro país. Es de destacar que fue el primero en la Argentina en utilizar ese término (Martínez Crovetto, 1964) en los títulos de trabajos de esa índole (Arenas, 1989).

Las publicaciones y cuadernos de trabajo de campo de Martínez Crovetto contienen múltiples datos y observaciones personales de valor científico, los cuales enriquecen la información sobre la etnobotánica general y regional de la época, dirigida tanto a los pueblos originarios como a los pobladores rurales en diversos puntos del país. Debe subrayarse que su mayor aporte lo hizo en la región del Nordeste argentino (NEA), motivado siempre por su interés en el gran acervo folklórico que ésta presenta (Krapovickas, 1989).

Por lo anterior, sus aportes revisten suma importancia en nuestro país, ya que logró implementar un nuevo marco para la realización de los estudios etnobotánicos en la Argentina. Toda la obra de Martínez Crovetto plasma su necesidad insaciable de conocimiento, quedando demostrado su perfil de gran investigador, curioso, inquieto, bibliógrafo; pero, sobre todos los aspectos, una persona comprometida y con gran sentido humano.

\section{De la Botánica a la Etnobotánica}

Como lo demuestra su currículo, Martínez Crovetto ha dedicado la mayor parte de su vida a temas relacionados a la botánica, publicando su primer trabajo científico referido al género Echinochloa en 1942 (Martínez Crovetto, 1942). A pesar de tener una extensa trayectoria en esta disciplina, se observa desde sus comienzos una gran motivación en realizar trabajos enfocados a la botánica aplicada. Hecho demostrado en sus primeros trabajos científicos, donde incluye a los vegetales en relación al uso que el hombre hace de ellos (Martínez Crovetto, 1944, 1947, 1948a).

Los motivos de este interés pueden ser variados, pero no debemos olvidar que una influencia importante habrá significado para él haber sido alumno, de las clases de botánica dictadas por el Ing. Agr. Lorenzo R. Parodi (Krapovickas, 1989). Además, su personalidad incluía un gran sentido humanista, como lo recuerdan hoy sus amigos (Schinini, com. pers.). A partir de estas diferentes motivaciones se acrecentó a lo largo de su carrera el interés por los estudios botánicos que incluyen a los seres humanos. De esta manera, logró integrar en sus investigaciones aspectos referidos a los vegetales, y lo que la gente hace con ellos, así como el sentido que le dan a lo que hacen. Un detalle no menor, se manifiesta en sus trabajos publicados a partir de 1963, en los cuales atiende aspectos referidos al uso, costumbre, identificación de fitónimos e, incluso, trabajos de tipo etnográfico sobre diferentes culturas de nuestro país.

A continuación, se transcribe una expresión personal manifestada en una de sus publicaciones, que sintetiza sus motivaciones por el quehacer etnobotánico en relación a su 
interés por consolidar la identidad nacional:

“...Es evidente que el pueblo guarda en su conocimiento un rico tesoro de sabiduría, acumulado y acrecentado con el devenir del tiempo y que su análisis y exposición razonada, deja establecido los lineamientos y el sentido de la ciencia popular, siendo de utilidad teórica, por cuanto para tener un sentimiento de lo nacional es necesario conocerlo -no se ama lo que se ignora- y practica..." (Martínez Crovetto, 1981a).

\section{Enfoques abordados dentro de la Etnobotánica}

- Etnobotánica histórica: revisión de documentos históricos e identificación y discusión sobre los taxones mencionados o sugeridos en ellos. Ej. Las noticias etnobotánicas de Augusto Guinnard (Martínez Crovetto, 1963).

- Generalista, cualitativo, inventarial: en sus trabajos de Estudios Etnobotánicos I a IV, realizados respectivamente con Tobas, Vilelas, Araucano Pampas, Onas (Martínez Crovetto, 1964, 1965, 1968a, 1968b).

-Análisis cuantitativos: a través de la comparación cuantitativa realizada entre las etnias del Nordeste Argentino (Martínez Crovetto, 1968c).

-Etnotaxonomía (recopilación y análisis de fitónimos), arquitectura de los fitónimos indígenas (NEA) (Martínez Crovetto, 1968c).

-Etnobotánica taxonómica: descripción y etnobotánica de nuevas especies usuales (Martinez Crovetto, 1948a, 1948b)

-Etnobotánica con enfoque etnográfico: alimentación, agricultura, magia (Martínez Crovetto, 1968, 1980a, 1980b).

- Estudios de malezas (Martínez Crovetto, 1944, 1946, 1957)

Por lo expuesto, podemos afirmar que Martínez Crovetto concibió los cimientos de la disciplina etnobotánica, en un marco de tolerancia entre los diferentes enfoques metodológicos y los diversos tópicos de interés, pues pudo complementar armoniosamente en su persona muchas de las perspectivas que hoy en día se encuentran representadas en la comunidad etnobotánica nacional.

\section{Aproximación al método empleado por Martínez Crovetto}

A pesar de haber caminado siempre por pasillos de aulas de ciencias agronómicas y exactas, escenarios con incontables miradas positivistas, no mermó su inquietud por realizar trabajos que tomen como punto de partida todo aquello que el ser humano es capaz de construir, conocer, y transformar. La intensidad de esta inquietud ha quedado impregnada en cada uno de los documentos que describen una amplia gama de interacciones, materiales y simbólicas, establecidas entre el hombre y el ambiente, las cuales han sido transmitidas desde la oralidad, a partir del conocimiento resguardado entre los lugareños, expresión cultural de los pueblos.

La mayoría de los manuscritos dejados por Martínez Crovetto consisten en redacciones que describen aspectos del objeto que se esta utilizando, siempre acompañadas de su designación en el idioma primario de la comunidad en estudio. Esta recurrencia en sus escritos podría indicar que su abordaje fue cualitativo, minuciosamente descriptivo e interpretativo. De esta manera, todo su trabajo se valió de esclarecer, a través de la transcripción de las palabras de los lugareños, cómo comprendían y ordenaban su universo natural circundante. Se destaca en sus textos la manera de evidenciar como ciertas plantas nos informan sobre las diferentes pautas culturales de acuerdo al contexto donde se insertan. Un ejemplo de estos trabajos son: "Plantas mágicas de los indios guaraníes de Misiones" (Martínez Crovetto, 1980) o "Plantas reguladoras de la fecundidad utilizadas en medicina popular del nordeste argentino" (Martínez Crovetto, 1981b).

En los archivos revisados, depositados en el Instituto de Botánica del Nordeste, no se encontraron modelos de entrevistas, ni cintas magnetofónicas. Sin embargo, según las anotaciones de sus cuadernos de campo, se puede divisar que se basaba en preguntas disparadoras, semejantes a las que hoy se implementan en las llamadas "entrevistas semiestructuradas", atendiendo de esta manera a las distintas circunstancias que aparecían en el momento del diálogo. Aún así, puntualiza mucho en las plantas medicinales, aunque no 
está documentado que haya tenido algún punto de interés en particular.

Se resalta en el trabajo etnobotánico de Martínez Crovetto la impronta de su formación botánica en la forma de organizar los datos, observándose que en la mayoría de sus escritos realiza inventarios botánicos, acompañados siempre de fitónimos y las diferentes formas de uso del vegetal en cuestión. Debe subrayarse que fue también uno de los primeros en resguardar la información recabada con ejemplares de herbario de referencia, los cuáles permitieron la identificación taxonómica más apropiada y la posterior documentación etnobotánica que hoy día se conserva el herbario CTES, del Instituto de Botánica del Nordeste (Fig. 1A).

\section{¿Cómo organizaba los datos?}

Se distingue el orden y la prolijidad con que organizaba sus datos de campo. Toda su obra demuestra una gran dedicación y organización personal, con la finalidad de que la información no se pierda.

En sus cuadernos se reconoce siempre la utilización de un sistema de clasificación en dónde definía diferentes categorías de usos, a través de las cuales organizaba los datos. Esta clasificación la realizó de manera jerárquica, en donde cada uno de los elementos que la integraban se establecían por relaciones de subordinación, cosubordinación o ambas clases de relaciones. Se entiende por subordinación la clasificación de conceptos como inferiores en orden con respecto a otros, y por cosubordinación la relación que se establece entre los miembros pertenecientes a un mismo nivel de jerarquía. Aún reconocido un sistema de categorización en sus cuadernos, se observa que al momento de redactar los manuscritos, estas categorías pueden superponerse, dependiendo del objetivo propuesto en el escrito.

\section{Martínez Crovetto y los juegos indígenas}

Muchos de sus trabajos de tipo etnográfico se dedican al estudio de los juegos en diferentes etnias de nuestro país. La relevancia de estos estudios, se debe al reflejo que demuestra la actividad lúdica como parte de la cultura popular, quedando a la vista diferentes aspectos, como las necesidades, las vivencias, las diferentes relaciones sociales y las establecidas con el ambiente.

En sus escritos, reconoció que hay juegos propios de cada etnia, y otros que se fueron renovando e, incluso, adquiriendo nuevos elementos y reglas a medida que se establecieron contactos con otros grupos étnicos.

En sus trabajos propone categorías de acuerdo a las finalidades de cada juego: azar, habilidad, recreación, deportivo e infantiles. Incluso algunos trabajos presentan un apartado sobre juguetes, donde el autor los describe como los demás juegos, a pesar de llevarse a cabo de manera individual.

La mayoría de los juegos estudiados se valen de piezas o elementos, materiales que pueden ser de origen vegetal o animal: de los 314 juegos documentados, 64 utilizan material vegetal y 28 de origen animal. También documentó elementos secundarios de los juegos como los premios y los amuletos.

\section{Folklore: música popular y yerba mate}

$\mathrm{Su}$ curiosidad y pasión en el patrimonio folklórico no sólo ha quedado plasmada en sus trabajos científicos; también la ha manifestado artísticamente, expresando sentimientos, circunstancias, vivencias, pensamientos e ideas presentes en el hombre a través de la música (Fig. 1B). Es así que ha compuesto una gran cantidad de canciones con diferentes estilos de música, con letras escritas por él mismo, donde pone de manifiesto el sentir y las costumbres del hombre, evidenciando una estrecha relación entre éste y su medio natural:

... Raíz de Taropé
traigo en un morral
para el corazón
inambú ca’á,
y si tiene tos
aromito en flor
tómelo en té,
es un buen pojá,
para el mal de amor
y contra el payé
pipi con clavel

... Raíz de Taropé

traigo en un morral

para el corazón

inambú ca'á,

y si tiene tos

aromito en flor

tómelo en té,

es un buen pojá,

para el mal de amor

pipi con clavel 


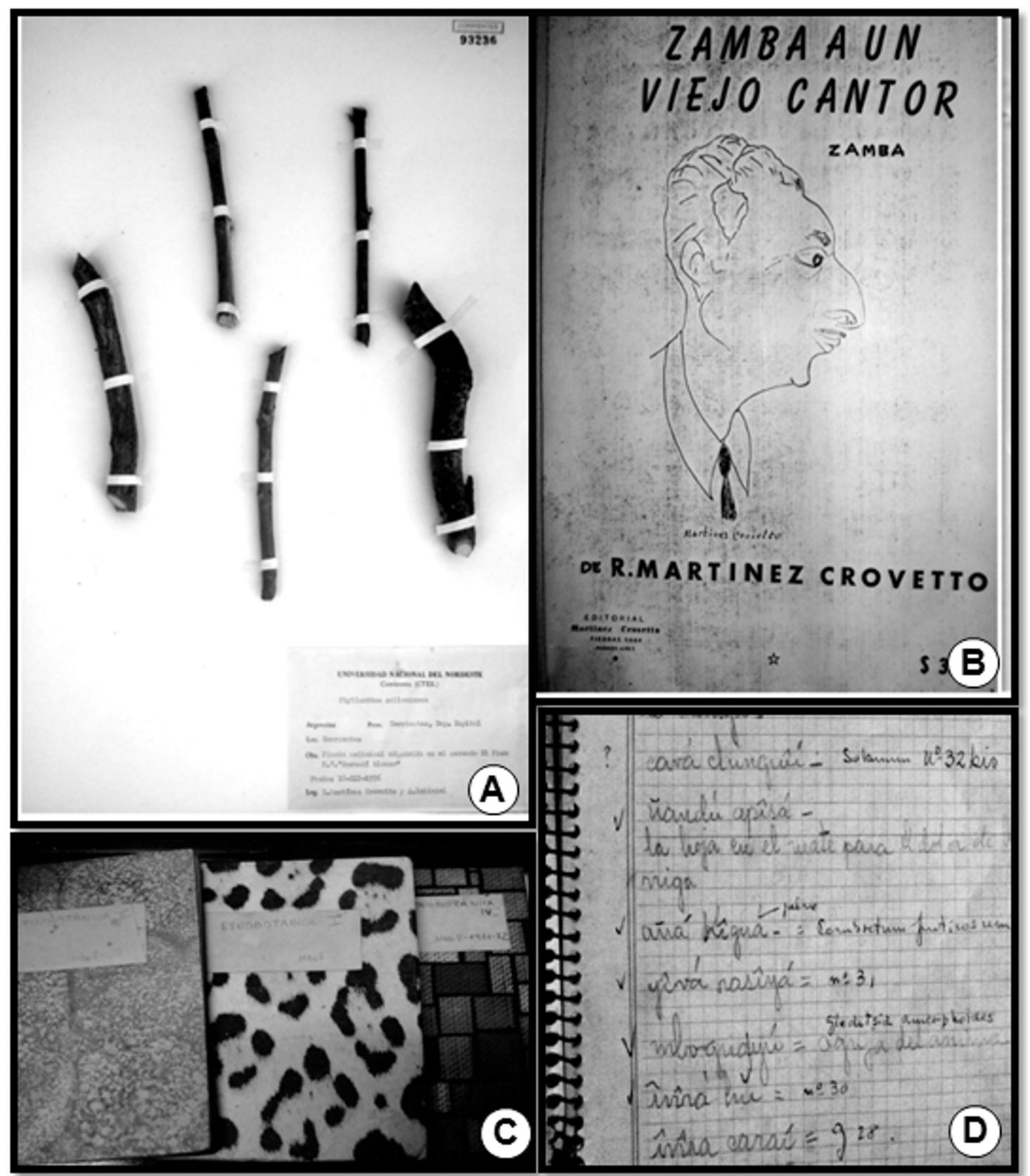

Fig. 1. A: Ejemplar de herbario de Martínez Crovetto depositado en CTES. B: Repertorio de zambas con letra y música de Martínez Crovetto, la obra fue editada por el autor y su tapa se ilustra con un autorretrato caricaturesco del mismo. C: Cuadernos de campo depositados en la biblioteca del IBONE. D: Notas de Martínez Crovetto.

mezclando los dos,

y para el riñón

parí-parobá

creame cuñá

eso es lo mejor...

Chamamé escrito y compuesto por el
Ing. Agr. Martínez Crovetto. Documentación hallada en la Biblioteca del Instituto de Botánica del Nordeste.

Dentro de su obra, ocupa un destacado espacio todo lo referido a la yerba mate, la bebida popular más difundida en nuestro país 
y en países aledaños. Si bien esta temática ha sido bien desarrollada por diferentes autores, lo que ha logrado Martínez Crovetto es colocar un importante acento en las cuestiones referidas a la historia y a la tradición popular de tomar mate. Se distingue en la recopilación de datos de sus trabajos de campo, distintos aportes paremiológicos:

“...A mate lavado, yerba buena, Proverbio misionero que enseña que, cuando algo no sirvió más, es mejor reemplazarlo, o que cuando una conversación o una instancia está agotada, es mejor cambiar de tema..." (Martínez Crovetto, 1995).

Iniciativas etnobiológicas y notas inéditas

Entre otras acciones que reflejan el interés de Martínez Crovetto por la relación sociedadnaturaleza, se destaca la fundación y edición de la revista "Etnobiológica", en 1967, bajo los auspicios de la Facultad de Agronomía y Veterinaria, Universidad Nacional del Nordeste. En esta se publicó un total de 14 números, donde aparecen muchos de sus trabajos, no sólo etnobotánicos, sino también etnográficos. Esta publicación fue efímera, como consecuencia de la falta de aceptación para su época (Arenas, 1989); a partir de 1970 se dejó de editar.

Entre los trabajos que publicó en esta revista se destacan las últimas dos contribuciones de la serie que denominó "Estudios Etnobotánicos I-IV" (Martínez Crovetto, 1968a,b), cuyas dos primeras entregas habían sido publicadas en Bonplandia (Martínez Crovetto, 1964, 1965).

$\mathrm{Su}$ trayectoria en Misiones y la amplitud de sus aportes referidos a los guaraníes nos conduce a pensar que el próximo documento de esta serie podría haberse denominado: Estudios etnobotánicos V. Nombres de plantas y su utilidad según los guaranies Mbya de Misiones. Probablemente, la elaboración de este hipotético manuscrito se haya visto truncada por su interés creciente en lo referido a los juegos indígenas, interés que dio a luz a las primeras cuatro publicaciones sobre el tema en 1968, coetáneas con la última contribución de la serie etnobotánica mencionada, y que siguió aportando artículos hasta el año 1979.

La biblioteca del Instituto de Botánica del Nordeste resguarda diversos materiales pertenecientes a Martínez Crovetto, que reflejan su impecable laboriosidad. Entre estos, se destacan varios cuadernos con recortes de diarios, microfilmes y notas de campo (Fig. 1C y D). Entre las notas que aparecen temáticamente ordenadas encontramos un gran cúmulo sobre fitónimos y usos de plantas provenientes de su trabajo de campo con los guaraníes Mbya de Misiones. En esta edición de Bonplandia, revista dirigida por Martínez Crovetto entre 1967 y 1987, hemos decidido dar a conocer dichas notas de campo. Nos hemos tomado la atribución de ordenarlas de acuerdo al formato de la serie que el forjador de la etnobotánica en la Argentina denominó: "Estudios Etnobotánicos".

\section{Agradecimientos}

Se agradece profundamente la asistencia de los bibliotecarios del Instituto de Botánica del Nordeste, y al Sr. Aurelio Schinini por los diversos relatos sobre la vida del Ing. Agr. Martínez Crovetto. Al Ing Agr. Krapovickas por la lectura del manuscrito y a los revisores quienes han mejorado la versión final.

\section{Bibliografía}

ALBUQUERQUE U.P. 1997.Etnobotânica: uma aproximação teórica e epistemológica. Rev. Bras. Farm. 78 (3): 60-64.

ARENAS, P. 1989. Homenaje RaúlNereo Martínez Crovetto (1921-1988): su contribución a la etnobotánica y a los estudios americanistas. Parodiana 5 (2): 505-519.

CABRERA, A. L. 1979. Evolución de las ciencias en la República Argentina 1923-1972. Tomo 6. Botánica. Sociedad Científica Argentina, Buenos Aires. 107 pp. DOMINGUEZ, J. A.1928. Contribución a la materia médica argentina. Trab. Inst. Bot. Farmac. Univ. Buenos Aires 44: 1-423.

FORD, R.1978. The nature and the status of ethnobotany. Anthropological Papers 67: 29-32.

HIERONYMUS, J. 1882. Planta Diaphoricae. Florae Argentinae. Bol. Acad. Nac. Ci, Córdoba 4 (2): 199598.

KRAPOVICKAS, A. 1989. Raúl N. Martínez Crovetto (1921-1988). Bonplandia 6 (2): 83-91.

MARTINEZ CROVETTO, R. N. 1942. Las Gramíneas argentinas del género Echinochloa. Rev. Argent. Agron. 9: 310-342.

- 1944. Las malezas de cultivo de papa en el sudeste 
de la provincia de Buenos Aires. Rev. Argent. Agron. 11 (3): 205-232.

1946. Dos malezas que no deben combatirse en los montes frutales silvestres de Misiones y Concordia. Almanaque Minist. Agric. 21: 85-87.

. 1948a. Los Solanum ornamentales cultivados en la República Argentina. Revista Invest. Agríc. 2 (4): 179-195.

. 1948b. Notas sobre plantas indígenas cultivadas en la Argentina. Revista Invest. Agric. 2 (3): 105-116.

- 1957. Plantas invasoras del cultivo del tabaco en el noroeste argentino. Revista Invest. Agric. 11 (2): 99-144.

1963. Las noticias etnobotánicas de Augusto Guinnard. Primer Congreso del Área Araucana Argentina, II: 29-41. Neuquén.

- 1964. Estudios Etnobotánicos I. Nombres de plantas y su utilidad según los indios tobas del este del Chaco. Bonplandia 1(4): 279-333.

- 1965. Estudios etnobotánicos II. Nombres de plantas y su utilidad según los indios vilelas del Chaco. Bonplandia 2 (1): 1-28.

. 1968a. Estudios etnobotánicos IV. Nombres de plantas y su utilidad según los indios onas de Tierra del Fuego. Etnobiológica 3: 1-20.

. 1968b. Estudios etnobotánicos III. Nombres de plantas y su utilidad según los indios araucano-pampas del oeste de Buenos Aires (República Argentina). Actas 37 Congreso Internacional de Americanistas 2: 603-624; y Etnobiológica 12: 1-24.

1968c. Introducción a la etnobotánica aborigen del Nordeste Argentino. Etnobiologica 11: 1-10 y en Actas del $38^{\circ}$ Congreso Internacional Americanista 3: $91-97$.
—. 1980. Plantas mágicas de los indios guaraníes de Misiones (República Argentina). La Antropología Americanista en la Actualidad 2: 447-454.

_. 1981a. Las plantas utilizadas en medicina popular en el Noroeste de Corrientes (Argentina). Miscelánea 69: 1-140. Inst. M. Lillo, Tucumán.

- 1981b. Plantas reguladoras de la fecundidad utilizadas en la medicina popular del nordeste argentino. Parodiana 1 (1): 97-117; y América Indígena 47 (2): 279-293.

- 1995. La yerba mate. Maravilla de América. Museo de Motivos Argentinos José Hernández, Buenos Aires. 224 pp.

MATOSO, E. 1893. Cien industrias, notas sobre plantas útiles escogidas de la flora correntina. Imprenta Llano, Corrientes. 337 pp.

PARODI, D. 1886. Notas sobre algunas plantas usuales del Paraguay, de Corrientes y de Misiones. Coni, Buenos Aires. 114 pp.

PARODI, L. R. 1934. Las plantas indígenas alimenticias cultivadas en la Argentina. Rev. Argent. Agron. 1: 65-212.

. 1935. Relaciones de la agricultura prehispánica con la agricultura argentina actual. Anales Acad. Nac. Agron. Vet. 1: 115-167.

—. 1961. Ciento cincuenta años de botánica en la República Argentina. Bol. Soc. Argent. Bot. 9: 1-68.

RAGONESE, A.E. \& R. N. MARTINEZ CROVETTO. 1947. Plantas indígenas de la Argentina con frutos y semillas comestibles. Rev. Invest. Agric. 1: 147-246

ROJAS ACOSTA, N. 1905. Plantas medicinales de Corrientes. Rev. Farm. 43 (45): 115-145.

- 1913. Essai d'une thérapeutique vegetale de Corrientes. Imprenta Dupuis, Resistencia 33pp.

Original recibido el 22 de mayo de 2012; aceptado el 12 de junio de 2012. 
